\section{Anestesia fuera del quirófano y COVID-19}

\author{
Out of operative room anesthesia and COVID-19
}

\author{
Dr. Eduardo Homero Ramírez-Segura,* Dr. Andrés De la Rosa-Mendoza ${ }^{\ddagger}$
}

RESUMEN. La demanda de apoyo de los servicios de anestesiología, fuera de las áreas «seguras» del quirófano, se ha ido incrementado exponencialmente, debido a la aplicación de nuevas técnicas y procedimientos diagnósticos, pronósticos, terapéuticos y paliativos. Estos procedimientos cada día más complejos, fuera de la sala de operaciones, ha dado pie a un capítulo más de la anestesiología, que hemos denominado «anestesia fuera de quirófano». Ante la pandemia de COVID-19, mucho se ha hablado de la reconversión de los hospitales y de los quirófanos, pero poco se ha hablado de la reconversión de las áreas y de las consideraciones en este tipo de procedimientos.

ABSTRACT. The high demand about anesthesiologist support out of the «security» operating rooms areas have increased exponentially, due to every day complex new practice in diagnoses, therapeutics and palliative technics. This proceedings, every day more complex have given rise to another chapter from the anesthesia, called anesthesia in non operating room area (NORA). In face the COVID-19 pandemic, there are a lot of papers about the restructuring of the operating rooms even the hospitals, but there is not enough information about NORA supports.

\section{El que no tiene conciencia clara de sus objetivos no sabe replicar al enemigo.} El arte de la guerra. Sun Tzu

$\mathrm{L}$ a «anestesia fuera de quirófano» (NORA, por sus siglas del inglés: nonoperatingroom anesthesia) es todo un capítulo más de la anestesiología, ya que se ha ido incrementando exponencialmente la demanda de apoyo de nuestros servicios fuera de las áreas «seguras» de quirófano. Esto se debe a los procedimientos cada día más complejos realizados fuera de las salas de operaciones como la aplicación de nuevas técnicas y procedimientos diagnósticos, pronósticos, terapéuticos y paliativos de las diferentes especialidades (Figura 1).

¿Por qué llamarlo fuera de las áreas «seguras» de quirófano? Porque los procedimientos anestésicos llevados a cabo en áreas apartadas e, incluso, lejanas al quirófano, pueden aumentar la morbilidad del paciente, ya sea por las bajas temperaturas a las que tienen que estar ciertos equipos, con riesgo de hipotermia para el paciente, o bien porque el personal de esas áreas está poco familiarizado con el quehacer del anestesiólogo y, muchas veces, es incapaz de apoyarlo ante una contingencia de la vía aérea, de una reacción anafiláctica, de un paro cardiorrespiratorio, de una broncoaspiración, etc., además de que, en muchas de las veces, falta el equipo de monitoreo, máquina de anestesia, aspiradores, fármacos, entre otros ${ }^{(1)}$; ahora, sumado a esto, ante la pandemia que estamos viviendo (por COVID-19), también falta equipo de protección personal (EPP) y hay poca atención que se le ha dado a la reconversión de esas áreas (Figura 2). No obstante, hay áreas de NORA en las que existe mucha conciencia de los riesgos de contagio y, al respecto, se están tomando todas las precauciones correspondientes, como en las salas de endoscopía, broncoscopía y radioneurocirugía, sólo por mencionar algunas (Figura 3).

Mucho se ha hablado y escrito también sobre la reconversión de los quirófanos (e incluso de los hospitales) a área COVID, pero poca atención se ha puesto a este rubro tan importante de la anestesiología, que es la NORA. Bajo el principio de precaución (adopción de medidas protectoras ante las sospechas fundadas de que ciertos eventos crean o provocan un riesgo grave para la salud pública o el medio ambiente), el personal sanitario (médi-
Anestesiología

Octubre-Diciembre 2020

Vol. 43. No. 4. pp 264-270

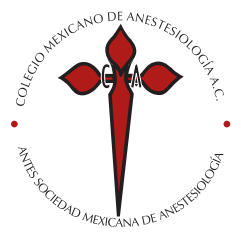

Palabras clave:

COVID-19, anestesia, quirófano, pandemia.

Keywords:

COVID-19, anesthesia, operating room, pandemic.

* Médico Naval, Anestesiólogo \& Neuroanestesiólogo. Academia Mexicana de Cirugía. Médico Adscrito Grupo Neuroquirúrgico Nave Medical. Exdirector de la Escuela de Posgrados en Sanidad Naval. Universidad Naval. Exjefe de División de Cirugía. Hospital Naval de Alta Especialidad. Secretaría de Marina-Armada de México. ‡ Médico Anestesiólogo Pediatra. Exjefe del Departamento de Anestesiología y Quirófanos del Instituto Nacional de Pediatría. SS. México. Anestesiólogo Adscrito. Unidad de Resonancia Magnética del Hospital Médica Sur. Fundación Clínica Medica Sur. México.

Solicitud de sobretiros: Eduardo Homero Ramírez Segura E-mail: ehramseg@gmail.com

Recibido para publicación: 13-05-2020

Aceptado para publicación: 08-07-2020 


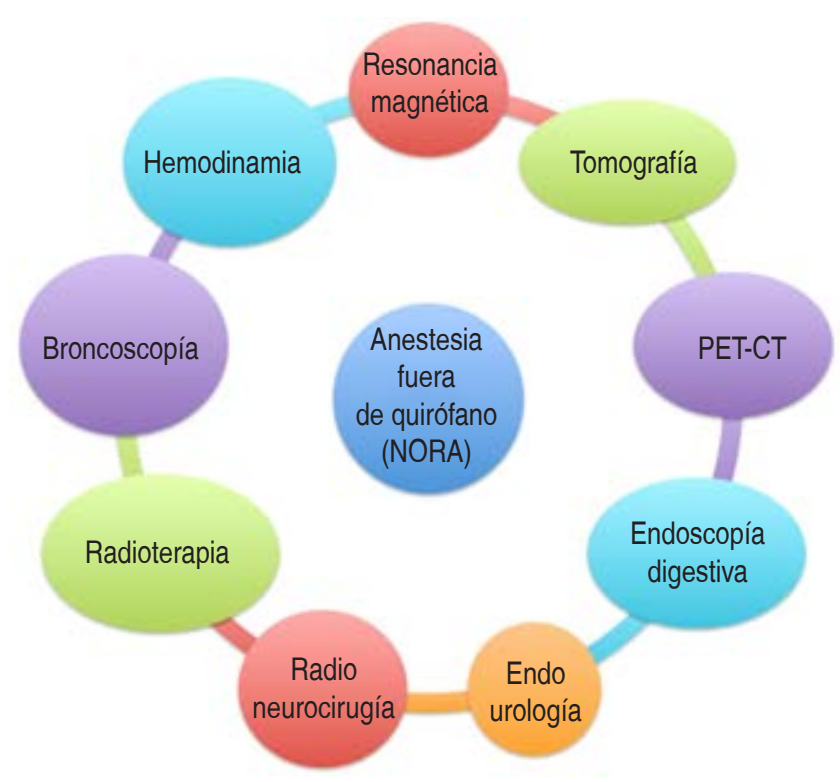

Figura 1: Anestesia fuera de quirófano (NORA).

cos, enfermería, técnicos, camilleros, afanadores, etc.) ha tomado conciencia de los riesgos, y poco a poco ha ido desarrollando la cultura del trabajo en equipo.

Ya es de todos conocido los protocolos, precauciones y procedimientos que se deben adoptar para el manejo y control de los pacientes y de la vía aérea, así como lo mínimo indispensable que debe contener el EPP. La protección del personal sanitario comienza con el apoyo de las autoridades administrativas de cada institución pública o privada: primero, proveyendo el equipo adecuado (EPP) a todo el personal en cualquier área donde preste sus servicios (incluidas aquéllas en donde se proporcione NORA); y segundo, haciendo una adecuada discriminación (triage) y programación de los procedimientos o estudios bajo el principio de categorizar adecuadamente lo urgente, lo prioritario y lo electivo ${ }^{(2,3)}$.

Prácticamente, todas las sociedades y colegios de las diferentes especialidades han publicado guías en las que definen qué padecimientos y procedimientos caen en los respectivos rubros de urgencias y electivos, sin exponer, por supuesto, al propio paciente ni al personal de salud. El médico anestesiólogo forma parte del personal sanitario con mayor riesgo de contagio ante esta pandemia, dado que es quien está más expuesto al manejo de la vía aérea, ya sea dentro de los quirófanos o fuera de ellos (NORA), e incluso en áreas de cuidados críticos en donde se solicita de su apoyo para asegurar y controlar la vía aérea. En la reconversión hospitalaria, está muy claro cuando un hospital es COVID y todo el personal sabe cómo debe conducirse y cuáles son las precauciones que debe tomar; en teoría, el personal se encuentra seguro con un adecuado EPP. Sin embargo, representa un peligro mayor cuando el hospital «no es COVID» y se corre el riesgo de que no se tomen las medidas de precaución correspondientes. Es crucial que tengamos muy presente que todos los pacientes se deben tomar como «portadores potenciales» y cumplir por esa razón con todos los protocolos de seguridad, como si se tratara de pacientes confirmados (Figura 4$)^{(4)}$.

La revista Time, en abril de 2020, publicó en su portada una imagen y un comentario que impactan; es el sentir de un médico anestesiólogo italiano (Francesco Menchise): «We must get it into our heads that our lives have changed» (Figura 5). Coincidiendo plenamente con él, que «la vida nos cambió y nos lo debemos meter en la cabeza», nunca imaginamos que para prestar nuestros servicios profesionales como anestesiólogos tuviéramos que emplear todo un EPP, incluso improvisando con equipo de grado no médico, pero siempre en aras de salvaguardar nuestra seguridad y en pro de que ningún paciente se quede sin atención oportuna (Figura 6). Por ello, es importante considerar que en las unidades en donde se lleve a cabo la NORA se cuente con todo el equipo necesario, como si de un quirófano se tratara, incluido el EPP que, de no contarse, se debe solicitar; además debe haber un compromiso institucional para que se provea.

En México, la Norma Oficial Mexicana (NOM-006SSA3-2011) para la Práctica de la Anestesiología ${ }^{(5)}$ previene que los establecimientos prestadores de servicios de atención médica y en los cuales se practique la anestesiología fuera de quirófano deben reunir un mínimo de requisitos en materia de equipamiento (Tabla 1).

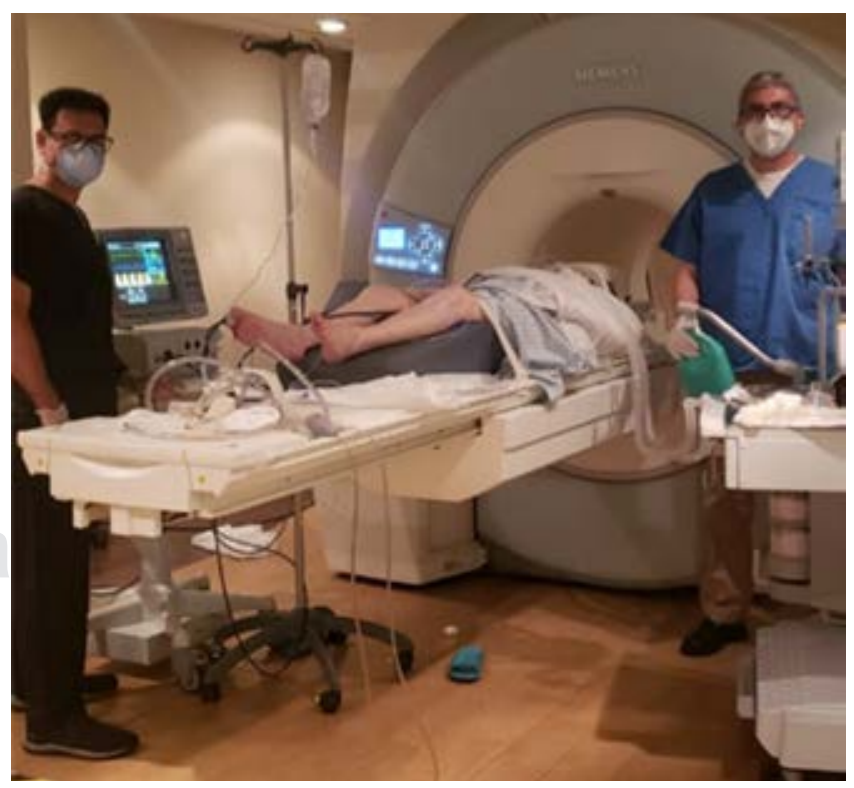

Figura 2: Unidad de resonancia magnética sin EPP. 


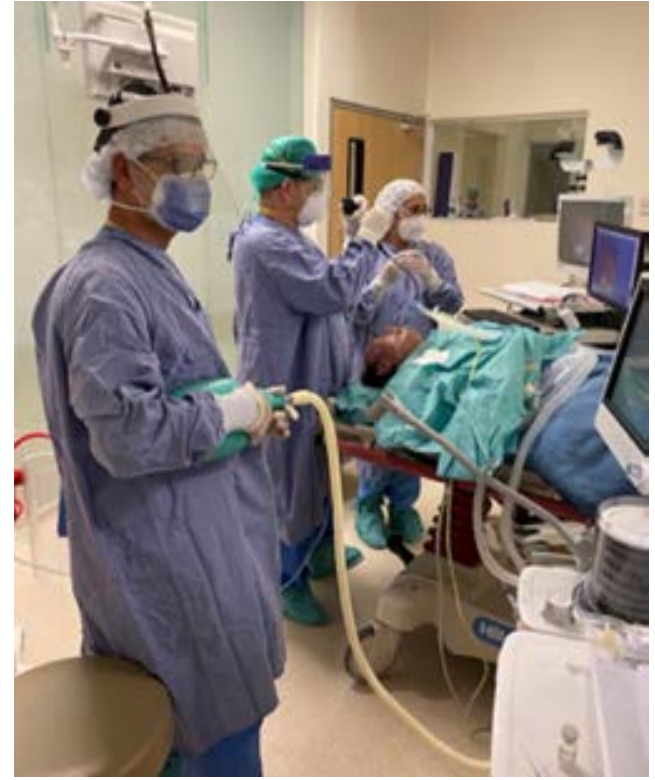

Figura 3: Sala de endoscopía.

\section{PERSONAL Y EQUIPO}

La anestesia fuera del quirófano requiere habilidades, experiencia, conocimiento y organización. La NOM para la Práctica de la Anestesiología define los requisitos que debe reunir el personal que proporciona la anestesia; si bien, la Norma no lo establece, debe ser personal que tenga experiencia, ya que la mayoría de las veces - como se comentó previamente-, el anestesiólogo está solo y el personal de esas áreas no está familiarizado con el quehacer de éste y no sabe cómo ayudarlo ante una contingencia, o bien ante la falta de personal o, incluso que no se le dé la adecuada importancia a la NORA, se envía al personal en formación, considerando los procedimientos NORA como «menores».

La preparación de la unidad (NORA) debe hacerse con los mismos estándares de calidad, tal como se hace en el quirófano; además, es necesario revisar y hacer la prueba de la máquina de anestesia. De no contarse con ella (por ejemplo, en México, pocos hospitales cuentan con una máquina de anestesia dentro de las unidades de resonancia magnética), se deberá asegurar una fuente de oxígeno, contar con un sistema para apoyo de la ventilación (como pudiera ser un sistema Bain), así como todos los dispositivos relacionados con la vía aérea (mascarillas faciales, cánulas endotraqueales, dispositivos supraglóticos, cánulas de Guedel, laringoscopio compatible, en caso de resonancia magnética con sus respectivas hojas), fármacos suficientes en cantidad y en variedad, un aspirador, además de que la unidad debe contar con un carro de paro ${ }^{(1)}$. Se debe realizar una lista de cotejo (checklist), previo a iniciar un procedimiento de NORA, teniendo siempre presente que, de requerir cualquier apoyo, éste tardará, dado que no es lo mismo que estar en el «área segura del quirófano».

\section{VALORACIÓN PREANESTÉSICA}

La valoración preanestésica para un paciente que va a ser sometido a un procedimiento bajo NORA debe hacerse con los mismos criterios que se utilizan cuando un

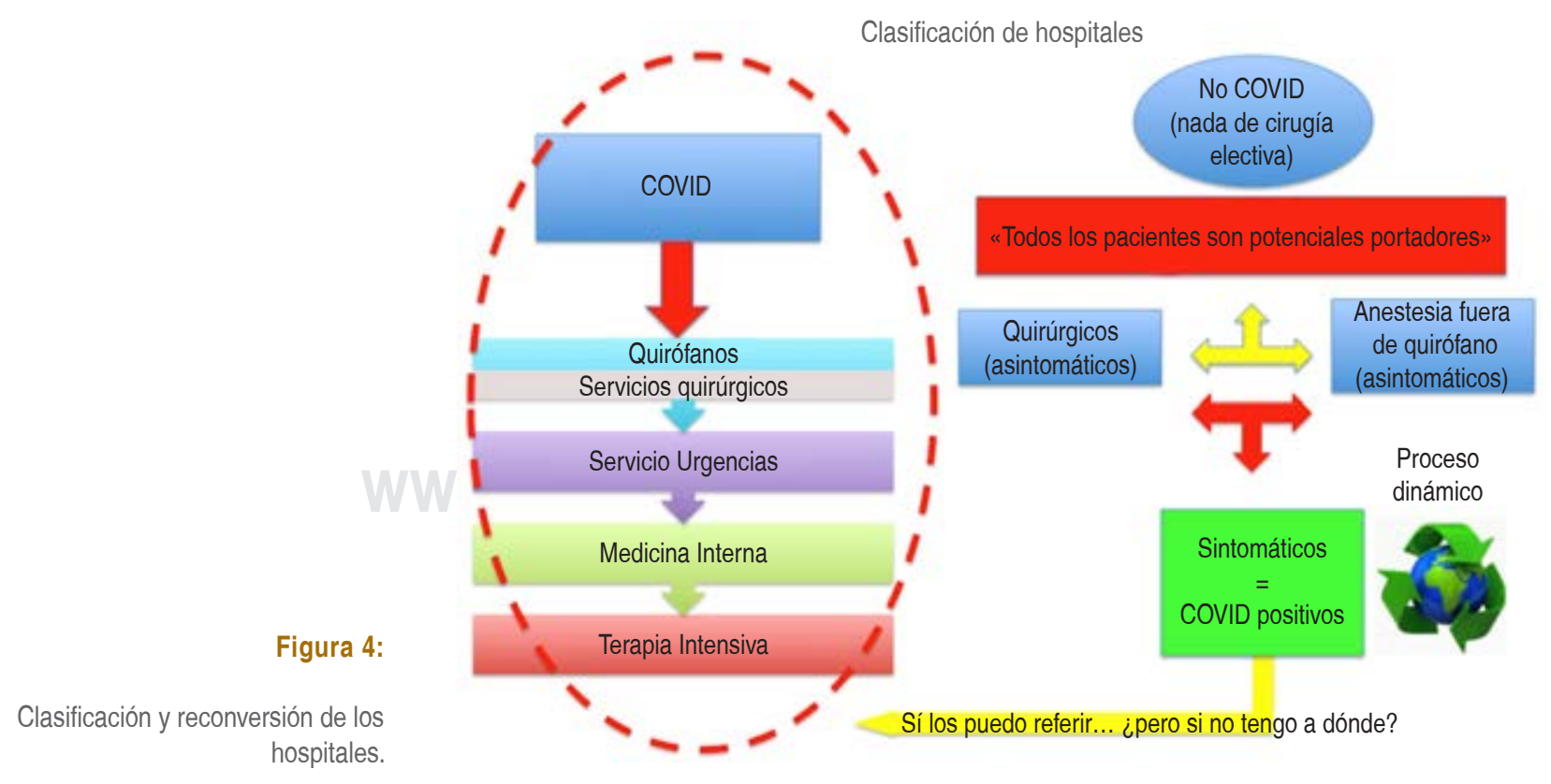




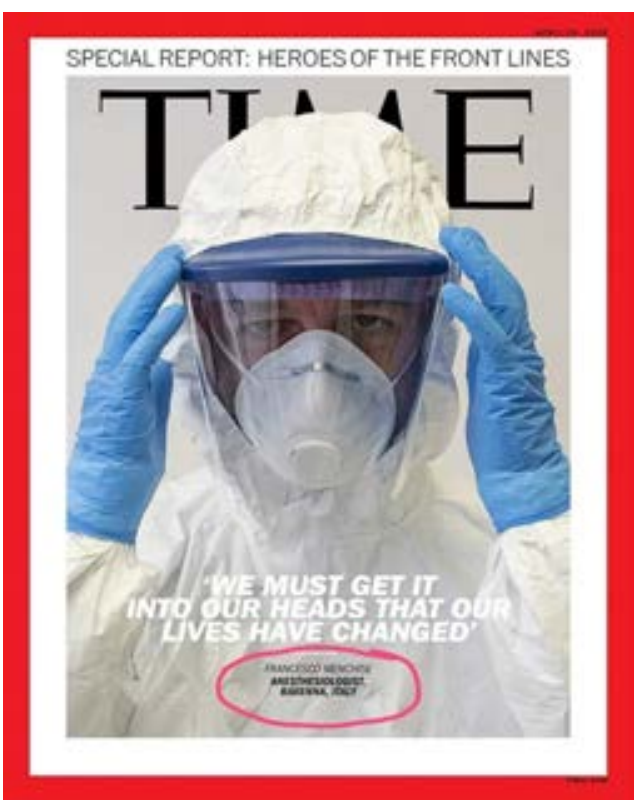

Figura 5: «Nos cambió la vida».

paciente va a ser intervenido quirúrgicamente, poniendo especial atención en los signos y síntomas sugerentes de la infección y haciendo hincapié en los antecedentes de posibles contactos con algún infectado. Debe planteársele al paciente o responsable legal de éste el plan de manejo, los riesgos, el tiempo de ayuno, según sea el caso, firmar el consentimiento informado (anexar los riesgos asociados con COVID-19) y se deberán solicitar los estudios de laboratorio apropiados para cada caso en particular (acordes con las necesidades del paciente, con el acto médico a realizar y con el protocolo de estudio respectivo). Actualmente, se debe incluir la prueba de COVID-19 con un máximo de 72 horas de vigencia. No está por demás informar que ciertas patologías implican la administración de material de con- traste para evidenciar la presencia de procesos isquémicos, inflamatorios o neoplásicos ${ }^{(1)}$.

Para el caso del paciente pediátrico, se ha determinado realizar la imagen de resonancia magnética (IRM) en aquellos pacientes en los que no es posible suspender la continuidad de su tratamiento (seguimiento de diagnósticos de epilepsia, postratamiento gamma knife y «estatus» convulsivo). Se debe realizar un interrogatorio a modo de «valoración preanestésica» vía telefónica, previamente a la programación; éste se corroborará el día del estudio. En dicho protocolo, se establece un esquema de ayuno de acuerdo con la edad del paciente. Se recomienda a los padres del paciente que programen el estudio a primera hora de la mañana para aprovechar la sanitización del equipo (contra

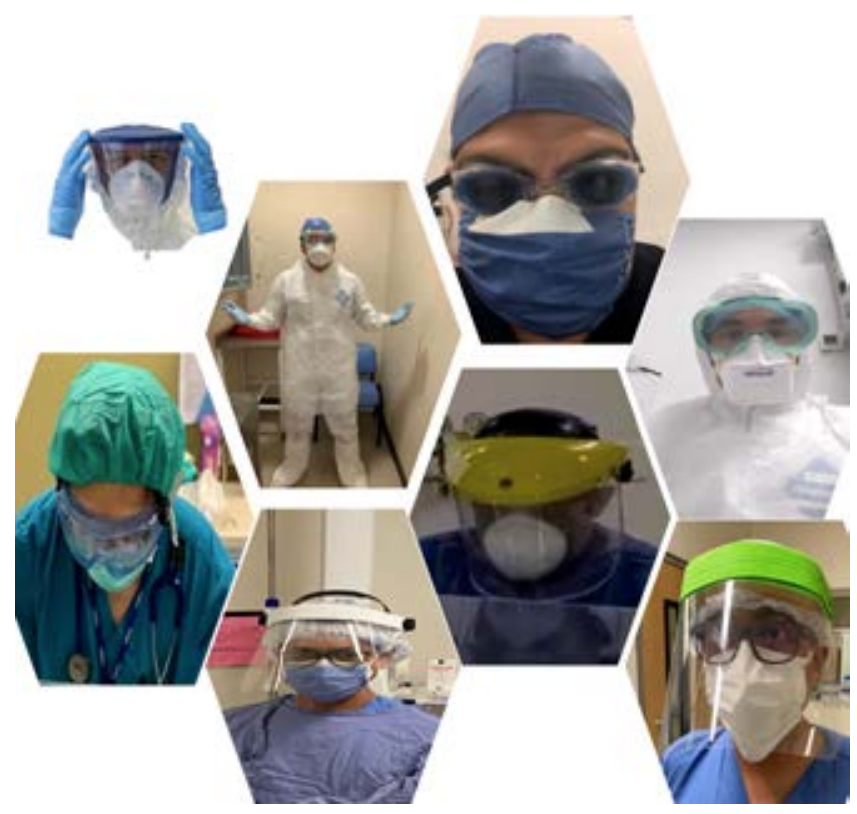

Figura 6: Reconversión del anestesiólogo.

Tabla 1: Apéndice B (normativo). Equipamiento para salas fuera del quirófano donde se realizan procedimientos anestésicos ${ }^{(5)}$.

\begin{tabular}{|c|c|c|}
\hline Concepto & $\begin{array}{l}\text { Establecimientos que } \\
\text { practican cirugía a adultos }\end{array}$ & $\begin{array}{l}\text { Establecimientos que } \\
\text { practican cirugía pediátrica }\end{array}$ \\
\hline Toma de oxígeno & & \\
\hline Equipo de aspiración & & \\
\hline Carro rojo para el manejo del paro cardiorrespiratorio & & \\
\hline $\begin{array}{l}\text { Máquina de anestesia en el caso de hemodinamia, tomografía axial computari- } \\
\text { zada y endoscopía }\end{array}$ & & \\
\hline Equipo de monitoreo especial para salas de resonancia magnética & & \\
\hline
\end{tabular}



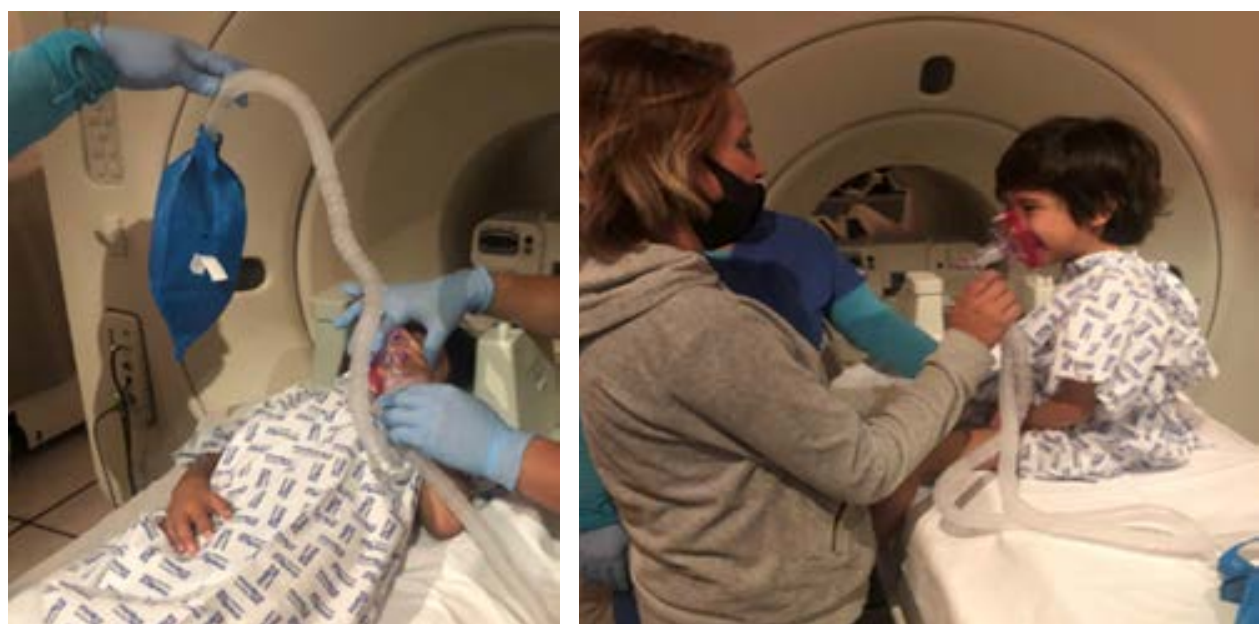

Figura 7:

Utilidad del circuito circular pediátrico modificado.
COVID-19), el cual se realiza en las primeras horas del día. Se indica diferir el estudio en caso de presentar fiebre, tos, gripe o diarrea, reprogramándose el estudio lo más pronto posible una vez ceda la sintomatología. El día del evento, deberá firmarse el consentimiento informado, especificándose además de los posibles riesgos anestésicos, el riesgo de contaminación por COVID-19.

\section{MANEJO ANESTÉSICO}

Recientemente, el Dr. Luciano Gattinoni (Anestesiólogo e Intensivista, Investigador Italiano, profesor de Anestesia y Cuidados Intensivos de las Universidades de Milán, Italia y Gotinga, Alemania) declaró que ante COVID-19: «... nadie tiene la verdad en esta enfermedad nueva. Aún no existen expertos sobre ella. Estamos aprendiendo». Ante estos acertados pronunciamientos, es muy complejo querer establecer manejos anestésicos en cualquier escenario clínico, incluido NORA. Al día de hoy, ya hay sociedades de anestesiólogos de diferentes países que han emitido protocolos con recomendaciones sobre NORA-COVID-19(4); no obstante, aún hay controversia en los manejos: si es mejor o no intubar a los pacientes por los riesgos de contagio por la aerolización al manejar la vía aérea, pero sobre todo en pacientes en los que se «comparte» la vía aérea con el procedimiento a realizar, como lo es una endoscopía de vías digestivas altas o una broncoscopía. También está la controversia o el criterio de intubar o no a un paciente COVID-19 positivo no grave, programado para una resonancia magnética o a un paciente asintomático. Pero ante la premisa de que «todo paciente es potencial portador» está la duda si intubamos o no en una resonancia magnética, partiendo de que, si se intuba se «cierra» el circuito con el inflado del manguito, el circuito anestésico y la máquina de anestesia; en caso de tenerla, no se expone al personal a la aerolización durante todo el procedimiento, sino sólo al mo- mento de la intubación y extubación. Otra controversia es si utilizar los dispositivos supraglóticos o cánula endotraqueal para el manejo de la vía aérea. Al respecto continuamos aprendiendo, pues aún no hay estudios con evidencia científica. Con base en la experiencia adquirida en estos seis meses de la pandemia, podríamos decir que sí hay evidencia de que la intubación de secuencia rápida es lo recomendable. El manejo farmacológico debe adecuarse de manera individualizada a cada escenario clínico al que nos enfrentemos, considerando la hemodinamia, el compromiso pulmonar, la respuesta inflamatoria sistémica, el comportamiento hematológico y, en general, el estado integral de cada paciente.

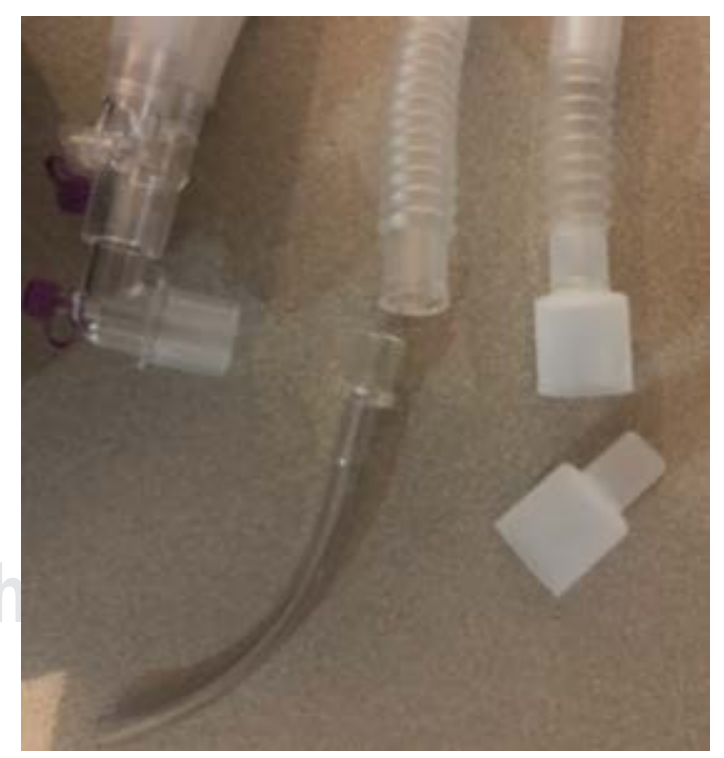

Figura 8: Deconstrucción del circuito circular al colocar un conector de sonda endotraqueal calibre 6.0 para la entrada de gases frescos (oxígeno/ sevoflurano). 
Para el caso del paciente pediátrico se ha establecido el manejo sin intubación endotraqueal, proporcionando anestesia general inhalatoria con mascarilla facial a través de un circuito circular pediátrico modificado (Figura 7). La modificación se realiza mediante la deconstrucción de un extremo del circuito, donde se inserta el conector de una sonda endotraqueal del calibre 6.0 recortada, para lo cual se dejará $10 \mathrm{~cm}$ de tubo para la conexión de gases frescos (Figura 8). La bolsa reservorio se modificará haciendo un pequeño orificio en el centro de la bolsa, el cual servirá de válvula espiratoria (Figura 9).

La modificación del circuito ofrece los siguientes beneficios: a) el paciente será inducido y mantenido bajo ventilación espontánea; b) separa del sistema de vaporización al paciente mediante un extremo de manguera de 2 a 3 metros, el cual suministrará los gases frescos; c) el anestesiólogo tiene en todo momento el control ventilatorio a través de mascarilla facial, que se mantiene fija en todo el evento de escaneo de resonancia; d) el circuito, al ser modificado en la entrada de gases frescos, permite el traslado a recuperación con oxígeno suplementario a través de tanques de traslado; e) conserva sanitizada la máquina de anestesia limitando su uso sólo para pacientes intubados que requieran ventilación mecánica (Figura 10).

Consideramos que el sevoflurano como medicamento único en niños es eficaz; asimismo, coincidimos con el Dr. Briggs $^{(6)}$, quien documentó los resultados de 5,864 casos para IRM en paciente pediátrico, con resultados óptimos en el $98.72 \%$ de los casos. A diferencia de ellos, nosotros realizamos una inducción inicial con 4-5 Vol\% de sevoflurano (en comparación, ellos iniciaron con $7 \mathrm{Vol} \%$ ) y mantenemos al paciente durante el estudio con 2 Vol\%; en la mayoría de los casos tratados hubo buenos resultados. La necesidad de intubar al paciente pediátrico en la IRM sólo se hace en casos de IRM de corazón y de abdomen en la que se requiere el manejo de apneas porque podemos instrumentarlas con el mismo sistema circular pediátrico modificado; sin embargo, estos casos son excepcionales, y en estos tiempos de COVID-19 no hemos tratado algún paciente pediátrico que requiera intubación endotraqueal en la IRM.

\section{CONSIDERACIONES GENERALES Y CONCLUSIONES}

El punto toral en la atención de pacientes infectados con COVID-19 que han sido confirmados, o bien, bajo la premisa de que «todo paciente es potencial portador» es garantizar la seguridad del personal sanitario que lo atiende y la propia seguridad del paciente, de modo que se debe contar con todos los recursos para una segura atención, como son instalaciones adecuadas, EPP (bata impermeable, guantes, gafas protectoras, careta, mascarilla N95, botas y gorro), capacitación en el uso de éste y en la manera de desecharlo, «aerobox» (uso controversial) y salas con presión negativa.

No obstante, es muy difícil que en procedimientos de NORA las salas cumplan este requisito. Por tanto, se debe realizar lavado de manos y cambio de ropa; además se
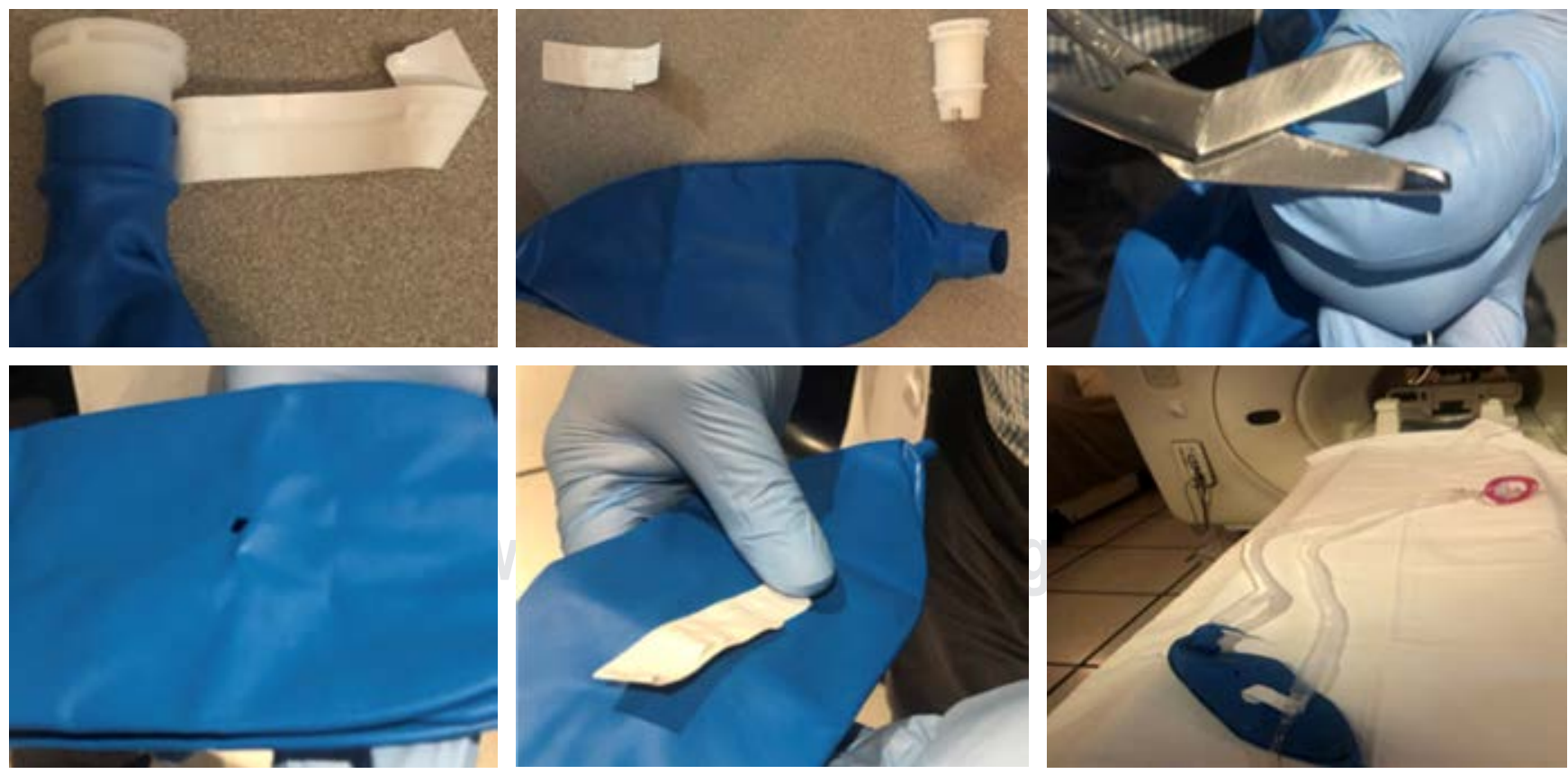

Figura 9: Deconstrucción de la bolsa reservorio con un pequeño orificio (válvula espiratoria) que se inserta en el extremo para cerrar el circuito circular. 
Figura 10:

Conservación del sanitizado de la máquina de anestesia en paciente pediátrico no COVID-19.
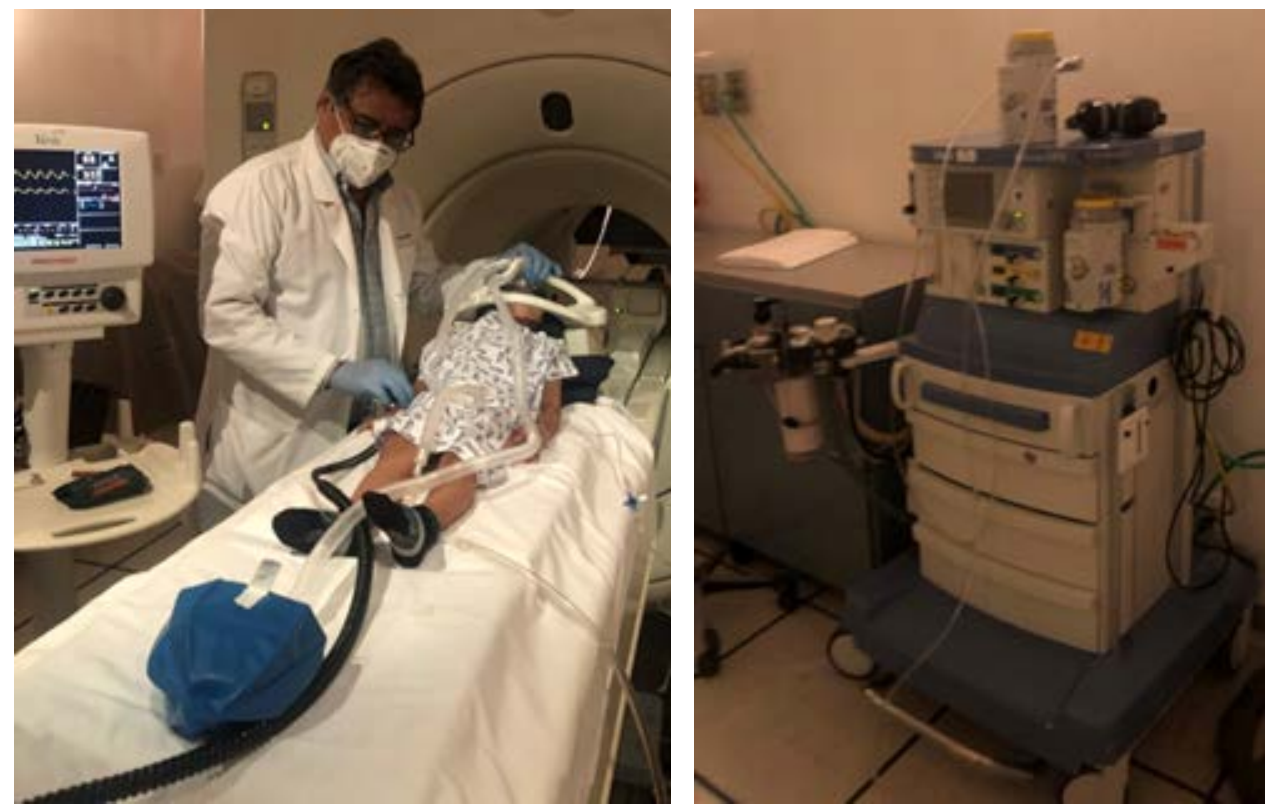

recomienda la menor concentración de personal sanitario que pueda participar en el procedimiento. También, se recomienda al menos la participación de dos anestesiólogos e incluso un tercero que se encuentre fuera de la sala y actúe como enlace. Como refiere Gattinoni, estamos aprendiendo; el tiempo y la evidencia científica nos irá marcando el rumbo y, entonces, ya podremos hacer un consenso global al respecto. Referencias

\section{REFERENCIAS}

1. Ramírez SE. Anestesia en resonancia magnética. En: E-PAC ${ }^{\circledR}$ Anestesiología-3. México: Ed. Intersistemas; 2014.

2. Portela-Ortiz JM, Ocampo-Valencia DBP. Anestesia en procedimientos endoscópicos en pacientes con SARS-COV-2/COVID-19. Rev Mex Anestesiol. 2020;43:197-198.

3. Pérezamador-del Cueto M. Implicaciones bioéticas en anestesiología durante la pandemia de COVID-19. Rev Mex Anestesiol, 2020;43:204205.
4. Recomendaciones para anestesia fuera de quirófano. Federación Argentina de Asociaciones de Anestesia, Analgesia y Reanimación. www.anestesia.org.ar

5. NOM-006-SSA3-2011, Para la práctica de la anestesiología. Diario Oficial de la Federación. Cuarta Sección. Marzo 232012.

6. Briggs V. Sedación con sevoflurano para resonancia magnética en pediatría: estudio clínico retrospectivo de 5.864 casos. Rev Esp Anestesiol Reanim. 2009;56:212-216. 